\title{
Sublingual sufentanil (Zalviso) patient-controlled analgesia after total knee arthroplasty: a retrospective comparison with oxycodone with or without dexamethasone
}

This article was published in the following Dove Press journal:

Journal of Pain Research

\section{David E van Veen' \\ Christiaan CWM Verhelst ${ }^{2}$ \\ Roelof T van Dellen ${ }^{3}$ \\ JSHA Koopman ${ }^{4}$}

'Erasmus University Rotterdam, Rotterdam, The Netherlands; 2Department of Orthopedic Surgery, Maasstad Hospital, Rotterdam, The Netherlands; ${ }^{3}$ Department of Hospital Pharmacy, Maasstad Hospital, Rotterdam, The Netherlands; ${ }^{4}$ Department of Anesthesiology, Maasstad Hospital, Rotterdam, The Netherlands
Correspondence: David E van Veen Department of Anesthesiology, Maasstad Hospital, Maasstadweg 2I, 3079 DZ Rotterdam, The Netherlands Tel +31 10291 19II

Email SublingualSufentanil@gmail.com

\begin{abstract}
Postoperative pain is a major problem, especially in orthopedic surgery. Our data suggest suboptimal pain management after total knee arthroplasty. This study evaluated a sufentanil sublingual tablet system (Zalviso) to optimize postoperative pain treatment. This retrospective, single-center, cohort study was conducted between January 2017 and September 2017. Zalviso as standard treatment was compared with a cohort receiving oxycodone (Oxy) immediate release and Oxy extended release and another receiving Oxy immediate release, Oxy extended release, and dexamethasone (Dexa + Oxy). The primary end point, pain intensity, was assessed on a numeric rating scale (NRS). Highest, lowest, and number of NRS scores $>7$ were collected. Secondary end points included length of hospital stay, nausea, and mobilization on the day of surgery. Patients receiving Dexa + Oxy had a lower lowest-pain intensity on day 0 (median 0 , IQR $0-0$ ) when compared to patients receiving Oxy (median 2, IQR 0-3; $P<0.0001$ ) or Zalviso (median 2, IQR 0-4; $P<0.0001$ ). No differences were observed on day 1 or 2 . No differences were observed in highest pain score or number of patients reporting NRS scores $>7$. Patients treated with Dexa + Oxy or Zalviso were discharged earlier compared to patients treated with Oxy $(P<0.001)$. Patients treated with Zalviso experienced more nausea compared to other groups on day 0 and day $1(P<0.001)$. Patients treated with Dexa + Oxy had a higher percentage of mobilization on the day of surgery compared to Oxy and Zalviso $(P<0.001)$. In conclusion, Zalviso did not improve postoperative pain management in patients undergoing total knee arthroplasty and increased nausea.
\end{abstract}

Keywords: total knee arthroplasty, postoperative pain, multimodal treatment, acute pain, Zalviso, sublingual sufentanil tablet system, opioid, sufentanil, sublingual formulation

\section{Introduction}

Total knee arthroplasty (TKA) is a commonly performed procedure, with over 700,000 joint replacements performed in the USA each year. ${ }^{1}$ In the USA, this procedure is projected to grow $>600 \%$ by 2030 compared to the level in 2005. Analysis of data for England and Wales also showed an expected increase in volume of primary TKAs in 2030 of $>100 \% .^{2}$ Despite increasing knowledge about postoperative pain management and the implementation of new painmanagement techniques, postoperative pain after TKA is still a major problem. ${ }^{3-7}$ Furthermore, TKA has turned to a fast-track approach, where early rehabilitation is a key factor in reducing morbidity and decreasing length of hospital stay. ${ }^{8,9}$ Our hospital data suggest suboptimal pain management after TKA, with around 10\% 
of patients reporting a numeric rating scale (NRS) scores $>7$ after surgery.

Patients with suboptimal pain management can experience chronic pain after surgery, increased length of hospital stay, less satisfaction, and decreased mobility, due to postoperative pain. ${ }^{10,11}$ Early mobilization is a key factor in improving postoperative recovery. In order to achieve early mobilization, postoperative pain management has to be optimized in such a way that patients experience minimal pain and have no restrictions, such as intravenous (IV) lines.

As a supplement to a multimodal analgesia regimen and in an effort to improve postoperative recovery, a sufentanil sublingual tablet system (SSTS; Zalviso) was introduced. Zalviso is a preprogrammed, noninvasive, patient-activated system that enables controlled analgesia without the need for an IV catheter. The device contains 40 sufentanil tablets (15 $\mu \mathrm{g}$ each) and uses a radiofrequency thumb-identification tag to allow only the patient to operate the device. The device has a 20-minute lockout interval to prevent overdosing. ${ }^{12-14}$ Multiple studies have shown that SSTS can be an effective patient-controlled pain-management modality after major orthopedic surgery. ${ }^{7,14-17}$

Zalviso was introduced in our hospital to optimize postoperative pain treatment in TKA, because our previous regime with oxycodone (Oxy) immediate release (IR) and Oxy extended release (ER) was inadequate. It was introduced in a test phase as standard treatment for 72 patients. After these 72 patients, we tested a group using Oxy IR, Oxy ER, and dexamethasone $40 \mathrm{mg}$ (Dexa + Oxy), since this was standard practice in another hospital. The objective of the current study was to evaluate the efficacy of Zalviso after TKA compared with two historical groups: a group using Oxy IR and ER with and without $4 \mathrm{mg}$ Dexa preoperatively.

\section{Methods}

This retrospective, single-center, cohort study was conducted at Maasstad Hospital in the Netherlands between January 2017 and September 2017. The study protocol was approved by the local ethics board of Maasstad Hospital. The board waived the requirement for written informed consent, since patient data confidentiality was maintained as all data were processed anonymously. This manuscript adheres to the applicable STROBE guidelines. ${ }^{18}$ This study was carried out in accordance with the principles of the Declaration of Helsinki. Patients were eligible for inclusion if they were scheduled to undergo TKA. Patients who could not use the Zalviso system (mental health, physical problems) and patients allergic to sufentanil were excluded. In practice, all consecutive patients were included.

Because of the inadequacy of conventional pain treatment, treatment with Zalviso was initiated in a test phase as standard treatment in 72 patients undergoing TKA. Since it was introduced as standard treatment in a predefined number of patients, no sample-size calculation was performed. After this period, treatment with Dexa + Oxy was initiated. Since patients were treated according to local protocol, all consecutive patients were treated similarly without any dropouts. As part of standard treatment, all patients received local infiltration anesthesia with ropivacaine $0.2 \%$, oral paracetamol 1 $\mathrm{g}$ four times daily, oral metamizole $1 \mathrm{~g}$ three times daily, IV droperidol $0.625 \mathrm{mg}$ up to four times daily as needed, and IV tranexamic acid $2 \mathrm{~g}$ preoperatively. Patients treated with oxycodone IR and oxycodone ER received oral oxycodone ER $10 \mathrm{mg}$ twice daily and oral oxycodone IR $10 \mathrm{mg}$ up to six times daily as needed. Patients treated with Dexa received IV Dexa $4 \mathrm{mg}$ preoperatively. As part of prophylactic antiemetic medication, almost all patients on general anesthesia received IV ondansetron $4 \mathrm{mg}$ during anesthesia and IV droperidol as rescue medication for the recovery ward.

Data were gathered from January 1, 2017 to the end of February 2017, when patients were treated with Oxy IR and Oxy ER. After March 1, 2017, patients were treated with Zalviso. From June 2017 onward, patients received Dexa + Oxy. During these periods, other procedures like operating technique remained largely the same. One item that changed was the early mobilization. In the Oxy cohort, patients were mobilized actively by a physiotherapist or nurse on day 1 . In the Zalviso and Dexa + Oxy, cohort patients were mobilized actively on day 0 . In the Dexa + Oxy group, early mobilization (day 0) was further optimized because of better availability of physiotherapy in evening hours.

Baseline characteristics were collected: age, sex, bodymass index, American Society of Anesthesiologists score, and method of anesthesia. The primary end point, pain intensity, was assessed on an NRS, where $0=$ no pain and $10=$ worst possible pain. Highest NRS, lowest NRS, and number NRS scores $>7$ were collected from all groups three times a day. When a patient reported an NRS $>7$, rescue medication was given, and 30 minutes afterward NRS was measured again. In this study, we reported NRS at rest. Secondary end points included length of hospital stay, nausea, and mobilization on the day of surgery. Data gathering was done uniformly by the same person (CCWMV) for all patients in all groups, since data were gathered as part of standard treatment. Patient satisfaction was available only for the Zalviso group, so no comparison could be made between groups. 


\section{Statistical analysis}

Analyses were based on an intent-to-treat approach. As some patients were discharged on day 1 and day 2 , pain scores and nausea analysis for these days were done without discharged patients. Data are presented as mean (SD) for normally distributed variables. Abnormally distributed variables are presented as medians (IQR). Analyses were done with the Kruskal-Wallis test for lowest and highest NRS scores and length of hospital stay. Dunn's post hoc test was used to confirm differences between these groups. NRS scores $>7$ were analyzed with Fisher's exact test. Nausea and mobilization on the day of surgery were analyzed with Pearson's $\chi^{2}$ test. A sensitivity analysis was performed in patients receiving only spinal anesthesia.

Bonferroni corrections were applied, since multiple tests were performed. Primary and secondary end points were considered statistically significant at $P$-values of 0.00556 and 0.01 , respectively. Bonferroni corrections were also applied when differences between groups were determined. Tables show $P$-values without Bonferroni correction. All statistical analyses were performed using SPSS version 25.0 (IBM Corporation, Armonk, NY, USA) and Stata/SE version 14.2 (StataCorp LP, College Station, TX, USA). $P<0.05$ was considered statistically significant.

\section{Results}

Of the 227 patients who had TKA, 68 patients received Oxy as postoperative pain management, 72 received Zalviso, and 87 received Dexa + Oxy. Baseline characteristics are displayed in Table 1. As can be seen, the groups were comparable regarding baseline characteristics. In the Zalviso group, eight patients dropped out for various reasons, such as a malfunctioning Zalviso (three patients), delirium (two patients), language problems (one patient), and two patients for reasons unknown. These dropouts were treated with oxycodone IR and oxycodone ER and were still analyzed in the Zalviso group.

\section{Pain scores}

Patients receiving Dexa + Oxy had statistically significant lower lowest-pain intensity on day $0(0$, IQR $0-0)$ when compared to patients receiving Oxy (2, IQR 0-3, $P<0.0001)$ or Zalviso (2, IQR 0-4, $P<0.0001$ ). On days 1 and 2, there were no differences in lowest pain scores. No difference in highest pain scores were observed on any day. No difference was observed between groups in patients reporting NRS $>7$ (Table 2, Figure 1). Patients treated with Oxy received 3.72 (SD 2.9) tablets oxycodone IR $10 \mathrm{mg}$ as rescue medication, those treated with Zalviso received 13.75 (SD 11.96) tablets sufentanil $15 \mu \mathrm{g}$, and those treated with Dexa + Oxy received 2.54 (SD 2.57) tablets oxycodone IR $10 \mathrm{mg}$ as rescue medication.

\section{Length of hospital stay}

Patients treated with Zalviso (2.0, IQR 2.0-3.0; $P<0.001$ ) and patients treated with Dexa + Oxy (2.0, IQR 2.0-3.0; $P<0.0001)$ were discharged earlier than patients treated with Oxy (3.0, IQR 2.0-3.5; Table 2).

\section{Nausea}

Patients treated with Zalviso experienced more nausea than other groups on day 0 (difference from Oxy 29\%, $P<0.001$; difference from Dexa + Oxy $33 \%, P<0.001)$ and day 1 (difference from Dexa + Oxy 20\%, $P=0.03$ ). No difference

Table I Demographics

\begin{tabular}{|c|c|c|c|c|c|}
\hline Variable & Oxy $(n=68)$ & $\begin{array}{l}\text { Zalviso } \\
(n=72)\end{array}$ & $\begin{array}{l}\text { Dexa + Oxy } \\
(n=87)\end{array}$ & $\begin{array}{l}\text { Total } \\
(n=227)\end{array}$ & $P$-value \\
\hline Age (years) & $67(49-92)$ & $68(43-86)$ & $69(42-90)$ & $68(42-92)$ & 0.67 \\
\hline Sex, n (\%) & & & & & 0.92 \\
\hline Male & $2 I(3 I)$ & $24(33)$ & $29(33)$ & $74(33)$ & \\
\hline Female & $47(69)$ & $48(67)$ & $58(67)$ & $153(67)$ & \\
\hline BMI, $\mathrm{kg} / \mathrm{m}^{2}$ (SD) & $31.5(5.5)$ & $30.5(5.1)$ & $31.2(5.3)$ & $31.1(5.3)$ & 0.48 \\
\hline ASA score (I/II/III) & $9 / 45 / 14$ & $3 / 53 / 16$ & $6 / 68 / 13$ & & 0.40 \\
\hline \multicolumn{6}{|l|}{ Anesthesia, n (\%) } \\
\hline Spinal & $58(85)$ & $66(92)$ & $73(84)$ & $197(87)$ & 0.32 \\
\hline General & $10(15)$ & $6(8)$ & $14(16)$ & $30(13)$ & \\
\hline \multicolumn{6}{|l|}{$\begin{array}{l}\text { Preoperative pain } \\
\text { medication, n (\%) }\end{array}$} \\
\hline Opioid & $10(15)$ & $17(24)$ & $17(20)$ & $44(19)$ & 0.41 \\
\hline Nonopioid & $31(46)$ & $32(44)$ & $35(40)$ & $98(43)$ & 0.77 \\
\hline
\end{tabular}

Notes: Analyzed with one-way ANOVA for continuous variables and $\chi^{2}$ for categorical variables.

Abbreviations: BMI, body-mass index; ASA, American Society of Anesthesiologists; Oxy, oxycodone (immediate release and extended release); Dexa, dexamethasone (4 mg); Dexa + Oxy, Dexa + preoperative immediate-release Oxy and postoperative extended-release oxycodone. 


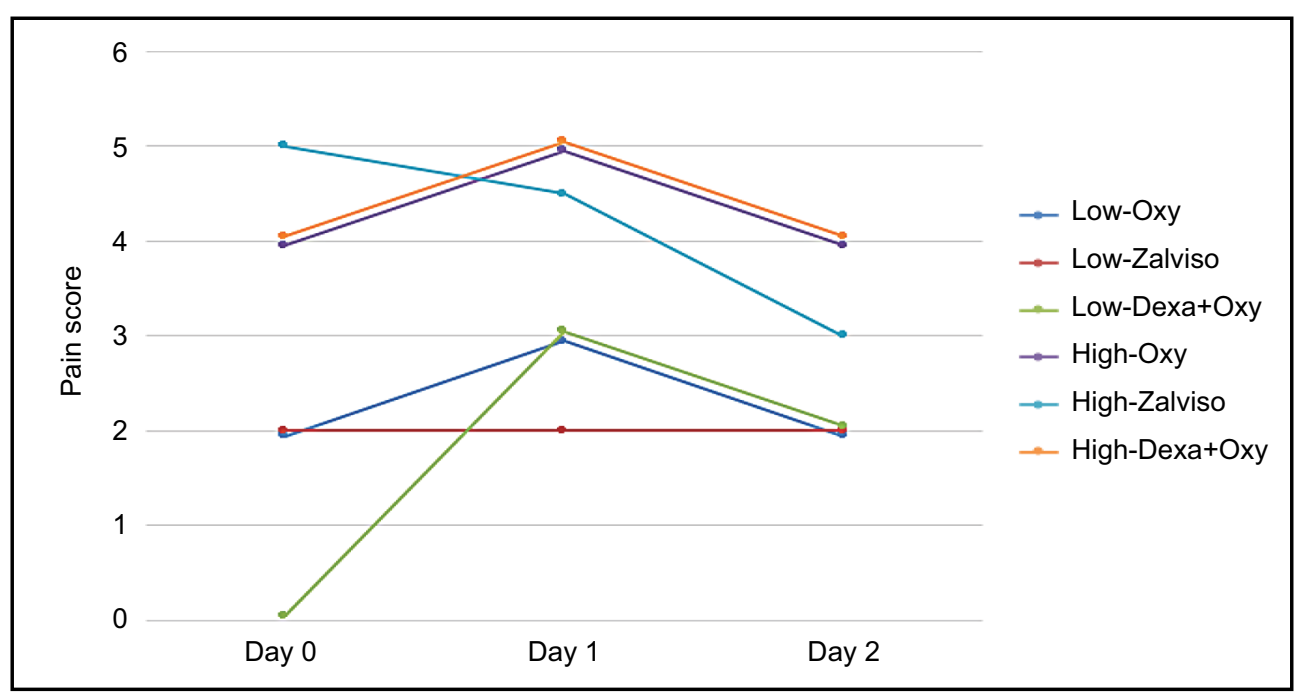

Figure I Lowest and highest pain scores, presented as medians.

Abbreviations: Oxy, oxycodone (immediate release and extended release); Dexa, dexamethasone (4 mg); Dexa + Oxy, Dexa + preoperative immediate-release Oxy and postoperative extended-release oxycodone.

Table 2 Pain scores, length of hospital stay, nausea, and mobilization on day of surgery

\begin{tabular}{|c|c|c|c|c|}
\hline Variable & Oxy & Zalviso & Dexa + Oxy & P-value \\
\hline \multicolumn{5}{|c|}{ Lowest pain score } \\
\hline Day 0 & $2(0-3)$ & $2(0-4)$ & $0(0-0)$ & $<0.0001$ \\
\hline Day I & $3(2-3)$ & $2(2-3)$ & $3(1-4)$ & 0.18 \\
\hline Day 2 & $2(I-3)$ & $2(1-3)$ & $2(2-4)$ & 0.10 \\
\hline \multicolumn{5}{|c|}{ Highest pain score } \\
\hline Day 0 & $4(4-5)$ & $5(4-6)$ & $4(4-5)$ & 0.24 \\
\hline Day I & $5(4-6)$ & $4.5(4-6)$ & $5(4-6)$ & 0.14 \\
\hline Day 2 & $4(3-4)$ & $3(2-4)$ & $4(2-4)$ & 0.25 \\
\hline \multicolumn{5}{|c|}{ NRS > 7, \% } \\
\hline Day 0 & $10.3(4.2-20.1)$ & $4.2(0.9-11.7$ & $4.6(1.3-11.4)$ & 0.27 \\
\hline Day I & $5.9(1.6-14.4)$ & $2.8(0.3-9.7)$ & $2.3(0.3-8.1)$ & 0.50 \\
\hline Day 2 & $3.1(0.4-10.7)$ & $1.6(0-8.7)$ & $0(0-4.9)$ & 0.30 \\
\hline \multicolumn{5}{|c|}{ Length of hospital stay, days } \\
\hline & $3.0(2.0-3.5)$ & $2.0(2.0-3.0)$ & $2.0(2.0-3.0)$ & $<0.001$ \\
\hline \multicolumn{5}{|l|}{ Nausea, \% } \\
\hline Day 0 & $22(13-34)$ & $5 I(39-63)$ & $18(\mathrm{II}-28)$ & $<0.001$ \\
\hline Day I & $46(34-58)$ & $51(39-63)$ & $31(22-42)$ & 0.03 \\
\hline Day 2 & $12(6-23)$ & $14(7-25)$ & $17(9-27)$ & 0.74 \\
\hline \multicolumn{5}{|c|}{ Mobilization on day of surgery, $\%$} \\
\hline & $35(24-48)$ & $63(50-74)$ & $85(76-92)$ & $<0.001$ \\
\hline
\end{tabular}

Notes: Lowest and highest pain scores presented as medians (IQR). NRS $>7$ presented as percentage of patients reporting NRS $>7$ ( $95 \%$ Cl). Length of hospital stay presented as medians (IQR). Nausea presented as percentages $(95 \% \mathrm{Cl})$. Since Bonferroni corrections applied, for primary and secondary end points, $P$-values of 0.00556 and 0.01 , respectively, were considered statistically significant. P-values shown here without Bonferroni correction. Primary end points included lowest NRS score, highest NRS score, and number of NRS scores $>7$. Secondary endpoints included length of hospital stay, nausea, and mobilization on the day of surgery.

Abbreviations: Oxy, oxycodone (immediate release and extended release); Dexa, dexamethasone (4 mg); Dexa + Oxy, Dexa + preoperative immediate-release Oxy and postoperative extended-release oxycodone; NRS, numeric rating scale.

between groups was observed on day 2 (Table 2). Results did not change when performing sensitivity analyses in patients receiving only spinal anesthesia.

\section{Mobilization on day of surgery}

Patients treated with Dexa + Oxy had a higher percentage of mobilization on the day of surgery compared to Oxy and 
Zalviso (difference from Oxy 50\%, $P<0.001$; difference from Zalviso $28 \%, P=0.003$ ). Patients treated with Zalviso had a higher percentage of mobilization compared to Oxy (difference 28\%, $P=0.003$; Table 2).

\section{Discussion}

In this study, we retrospectively compared three operative pain-treatment regiments for patients undergoing TKA. Based on our data, patients treated with Zalviso had more nausea than patients receiving Oxy and Dexa + Oxy without improving postoperative pain. The increased nausea was most likely the effect of sublingual sufentanil, and is in accordance with the literature. ${ }^{7,15}$ Pain scores were largely comparable between groups, except for day 0 , which showed better pain treatment in the Dexa + Oxy group. Although there was a statistically significant effect, one could argue that it was not clinically relevant.

Currently, pain management after TKA has shifted to a multimodal analgesic in a fast-track protocol to minimize opioid consumption and its adverse effects. ${ }^{19}$ Recent studies have acknowledged femoral nerve block as the most effective in TKA compared to other standard techniques, such as epidural, local infiltration anesthetic, and patient-controlled analgesia alone. ${ }^{20-22}$ Unfortunately, femoral nerve block adversely effects motor function, limiting early mobilization. ${ }^{20}$ To enhance early mobilization, multimodal oral pain treatment is combined with local infiltration anesthetic to balance optimal pain treatment with early mobilization. The current practice in our hospital reflects treatment advised in the literature. ${ }^{19}$

This study showed better mobilization on the day of surgery in Zalviso and Dexa + Oxy compared to Oxy. This could have been an effect of better availability of physiotherapy during this period. To improve mobilization, physiotherapy was increased to mobilize patients on the day of operation. This change was made at the start of Zalviso treatment and optimized in the Dexa + Oxy group. Lower pain scores on the day of operation could be explained by early mobilization and not be related to pain treatment. Definite conclusions cannot be drawn, given the nature of this study. Patients receiving Zalviso reported nausea more as the main reason for not mobilizing (10.0\% Zalviso vs 3.4\% Dexa + Oxy). Unfortunately, patient satisfaction could not be compared between groups, because we lacked data on patient satisfaction in the Dexa + Oxy and Oxy groups. Furthermore, since we tested Zalviso as part of standard treatment and the outcome of the Oxy + Dexa group was satisfactory, we had no possibility to test Zalviso + Dexa in our hospital. Adding a Zalviso + Dexa group might have shown additional benefit over the
Oxy + Dexa group. Future research is needed to provide a definite answer.

Apart from effectiveness, costs are a major concern. In our hospital, the use of Zalviso costs around $€ 100$ (US\$125) per patient when treating 300 patients a year. Furthermore, the cost of the device is around $€ 1,400$ $(\$ 1,750)$. Oxycodone is around $€ 0.60$ per tablet $(\$ 0.75)$. Since pain scores were similar between groups and given the nausea associated with Zalviso, Zalviso use was discontinued in our hospital.

In perfect circumstances, it takes the nurse 4 minutes to provide oxycodone to patients. Half an hour after administration, another NRS score was requested (30 seconds). Since patients using Zalviso have no need for oxycodone IR or oxycodone ER, it could save time at the ward. Replacing a thumb patch took about 2 minutes, which frequently occurred. That could have been why questionnaires showed only 9 of 17 nurses reporting a time-saving component when using Zalviso.

One might argue that patients undergoing general anesthesia could experience more nausea compared to patients undergoing spinal anesthesia. Since no difference was observed among the three cohorts regarding the number of patients with either anesthesia technique (general and spinal anesthesia), no bias is expected regarding nausea. A sensitivity analysis in patients receiving spinal anesthesia showed no differences.

In conclusion, Zalviso did not improve postoperative pain management in patients undergoing TKA and increased nausea compared to patients receiving Oxy and Dexa + Oxy. Patients receiving $4 \mathrm{mg}$ Dexa preoperatively and oxycodone IR and ER postoperatively were discharged earlier and had less nausea compared to all other groups. Given the costs associated with Zalviso, our study does not justify the use of Zalviso for postoperative pain treatment of patient undergoing TKA. To give a conclusive answer, a randomized controlled trial might be needed. Since this study only evaluated results directly after surgery, future studies could be expanded with long-term results and preoperative-pain scores, and furthermore the inclusion of more patients could help to get significant results. Since patients receiving 4 mg Dexa preoperatively showed improved outcomes, future studies might include adding Dexa to Zalviso treatment.

\section{Author contributions}

DEVV helped with writing the manuscript and data analysis, CCWMV helped with patient recruitment and data col- 
lection and conducted the Zalviso questionnaires among nurses, RTVD and JSHAK helped with the study design and implementation of Zalviso, and JSHAK also helped with directing the study project. All authors contributed toward data analysis, drafting and critically revising the paper, gave final approval of the version to be published, and agreed to be accountable for all aspects of the work.

\section{Disclosure}

The authors report no conflicts of interest in this work.

\section{References}

1. Kurtz S, Ong K, Lau E, Mowat F, Halpern M. Projections of primary and revision hip and knee arthroplasty in the United States from 2005 to 2030. J Bone Joint Surg Am. 2007;89(4):780-785.

2. Patel A, Pavlou G, Mújica-Mota RE, Toms AD. The epidemiology of revision total knee and hip arthroplasty in England and Wales: a comparative analysis with projections for the United States. A study using the National Joint Registry dataset. Bone Joint J. 2015;97-B(8):1076-1081.

3. Maheshwari AV, Blum YC, Shekhar L, Ranawat AS, Ranawat CS. Multimodal pain management after total hip and knee arthroplasty at the Ranawat Orthopaedic Center. Clin Orthop Relat Res. 2009;467(6): 1418-1423.

4. Xing Q, Dai W, Zhao D, Wu J, Huang C, Zhao Y. Adductor canal block with local infiltrative analgesia compared with local infiltrate analgesia for pain control after total knee arthroplasty: a meta-analysis of randomized controlled trials. Medicine (Baltimore). 2017;96(38):e8103.

5. Zhu Y, Feng Y, Peng L. Effect of transcutaneous electrical nerve stimulation for pain control after total knee arthroplasty: a systematic review and meta-analysis. J Rehabil Med. 2017;49(9):700-704.

6. Gan TJ, Habib AS, Miller TE, White W, Apfelbaum JL. Incidence, patient satisfaction, and perceptions of post-surgical pain: results from a US national survey. Curr Med Res Opin. 2014;30(1):149-160.

7. Jove M, Griffin DW, Minkowitz HS, Ben-David B, Evashenk MA, Palmer PP. Sufentanil sublingual tablet system for the management of postoperative pain after knee or hip arthroplasty: a randomized, placebo-controlled study. Anesthesiology. 2015;123(2):434-443.

8. Kehlet H, Thienpont E. Fast-track knee arthroplasty -- status and future challenges. Knee. 2013;20 Suppl 1:S29-S33.
9. den Hertog A, Gliesche K, Timm J, Mühlbauer B, Zebrowski S. Pathway-controlled fast-track rehabilitation after total knee arthroplasty: a randomized prospective clinical study evaluating the recovery pattern, drug consumption, and length of stay. Arch Orthop Trauma Surg. 2012;132(8):1153-1163.

10. Kehlet H, Jensen TS, Woolf CJ. Persistent postsurgical pain: risk factors and prevention. Lancet. 2006;367(9522):1618-1625.

11. Bugada D, Allegri M, Gemma M, et al. Effects of anaesthesia and analgesia on long-term outcome after total knee replacement: A prospective, observational, multicentre study. Eur J Anaesthesiol. 2017;34(10):665-672.

12. Minkowitz HS, Singla NK, Evashenk MA, et al. Pharmacokinetics of sublingual sufentanil tablets and efficacy and safety in the management of postoperative pain. Reg Anesth Pain Med. 2013;38(2):131-139.

13. Sacerdote P, Coluzzi F, Fanelli A. Sublingual sufentanil, a new opportunity for the improvement of postoperative pain management in Italy. Eur Rev Med Pharmacol Sci. 2016;20(7):1411-1422.

14. Melson TI, Boyer DL, Minkowitz HS, et al. Sufentanil sublingual tablet system vs. intravenous patient-controlled analgesia with morphine for postoperative pain control: a randomized, active-comparator trial. Pain Pract. 2014;14(8):679-688.

15. Meijer F, Cornelissen P, Sie C, et al. Sublingual sufentanil for postoperative pain relief: first clinical experiences. J Pain Res. 2018;11:987-992.

16. Morlion B, Schäfer M, Betteridge N, Kalso E. Non-invasive patientcontrolled analgesia in the management of acute postoperative pain in the hospital setting. Curr Med Res Opin. 2018;34(7):1179-1186.

17. Scardino M, D'Amato T, Martorelli F, et al. Sublingual sufentanil tablet system Zalviso for postoperative analgesia after knee replacement in fast track surgery: a pilot observational study. J Exp Orthop. 2018;5(1):8.

18. University of Bern IoSaPM. STROBE Statement Switzerland; 2009. Available from: www.strobe-statement.org. Accessed February 1, 2018.

19. Moucha CS, Weiser MC, Levin EJ. Current strategies in anesthesia and analgesia for total knee arthroplasty. J Am Acad Orthop Surg. 2016;24(2):60-73.

20. Albrecht E, Guyen O, Jacot-Guillarmod A, Kirkham KR. The analgesic efficacy of local infiltration analgesia vs femoral nerve block after total knee arthroplasty: a systematic review and meta-analysis. Br JAnaesth . 2016;116(5):597-609.

21. Chan EY, Fransen M, Parker DA, Assam PN, Chua N. Femoral nerve blocks for acute postoperative pain after knee replacement surgery. Cochrane Database Syst Rev. 2014;5:CD009941.

22. Ilfeld BM. Continuous peripheral nerve blocks: an update of the published evidence and comparison with novel, alternative analgesic modalities. Anesth Analg. 2017;124(1):308-335.
Journal of Pain Research

\section{Publish your work in this journal}

The Journal of Pain Research is an international, peer reviewed, open access, online journal that welcomes laboratory and clinical findings in the fields of pain research and the prevention and management of pain. Original research, reviews, symposium reports, hypothesis formation and commentaries are all considered for publication.

\section{Dovepress}

The manuscript management system is completely online and includes a very quick and fair peer-review system, which is all easy to use. Visit http://www.dovepress.com/testimonials.php to read real quotes from published authors. 